\title{
MRI of the Pancreas
}

MRI provides comprehensive information on the full range of pancreatic diseases. We employ a set protocol incorporating various types of sequences including transverse and coronal data acquisitions, and with the routine use of intravenous gadolinium.

\section{IMAGING THE PANCREAS}

The high signal-to-noise ratio (SNR) obtained at high field strength makes it possible to image the pancreas during a single breath-hold sequence. The sequences described herein are based on the authors' experience with a Siemens 1.5 T Vision scanner, but are expected to be equally applicable to machines from other manufacturers.

Scanning a patient or volunteer is a joint effort among technologists, nurses, and physicians, with the technologist normally responsible for following proper scanning protocols and techniques. Unless otherwise specified, in what follows, the person to whom directions are given is assumed to be the technologist. Table A18.1.1 lists the hardware necessary to perform the procedure, along with appropriate parameters.

The following ten sequences comprise the pancreatic imaging protocol. Most sequences require the patient to be able to suspend respiration for $\sim 20 \mathrm{sec}$. It is imperative that there be clear communication between the technologist and the patient throughout the exam. This protocol results in consistent, reproducible image quality that is effective for evaluating the full spectrum of pancreatic diseases.

NOTE: Be sure that technologists and nurses have immediate access to any emergency equipment that may be relevant to a given study, or that may be needed for a particular patient, such as crash carts or oxygen.

\section{Materials}

Normal saline $(0.9 \% \mathrm{NaCl})$, sterile, $40 \mathrm{ml}$ minimum

Extravascular contrast agent (e.g., Magnevist, Omniscan, or Prohance), volume prescribed by patient weight

\section{Set up equipment and patient}

1. Interview (screen) the patient to assess for contraindications such as cardiac pacemaker, implanted mechanical devices, and/or ferromagnetic materials. Also, determine if the patient has any health conditions that may require the presence of special

Table A18.1.1 Equipment Specifications: Imaging of the Pancreas

\begin{tabular}{|c|c|}
\hline Coil type & Circularly polarized body phased array coil \\
\hline Manufacturer and system type & Siemens Vision \\
\hline Field strength & $1.5 \mathrm{~T}$ \\
\hline Gradient strength & $\begin{array}{l}24 \mathrm{mT} / \mathrm{m} \text { (or whatever the system permits, } \\
\text { but minimum of } 24 \mathrm{mg} / \mathrm{m} \text { for sequences } 3 \\
\text { and } 4 \text { ) }\end{array}$ \\
\hline Knee cushion & Yes \\
\hline Use of contrast agents & Yes \\
\hline Pulse oximeter & If patient requires sedation \\
\hline Power injector & Yes \\
\hline Normal saline & Yes \\
\hline 35-in. extension tubing & Yes \\
\hline
\end{tabular}

BASIC PROTOCOL 
emergency equipment during the scanning procedure, or if he or she will need sedation medication necessitating the use of appropriate monitoring equipment.

Generally standard screening forms are used for all patients scanned in a magnetic resonance system.

The presence of any ferromagnetic metals may be a health hazard to the patient when he or she is inside the magnet, and will also affect the imaging. If in doubt as to the exact composition of the items, it is best to exclude patients with any metal implants; see Shellock (1996) for discussion of what implants may be safely scanned using magnetic resonance. The presence offerromagnetic materials in the globe of the eye is contraindicated for MRI. Patients with prior metal exposure to the eye should have plain $X$ rays of the orbital area to ensure that all metal has been removed prior to placing them in the magnetic field.

Patients may be accompanied into the magnet room by a friend or family member, who can sit in the room during the scan and comfort the patient as needed. This companion must be screened as well to ensure the absence of loose metal objects on the body or clothing.

2. Request that the patient change into a gown to eliminate any metal that might be found in clothing. Ask the patient to remove all personal effects such as, jewelry, hearing aids, glasses, etc., prior to entering the MRI scan room.

All personal belongings should be secured during the examination.

3. Explain the procedure to the patient and record relevant clinical history. Ensure that the patient understands what is expected and ask them if they have any questions; answer appropriately. If the procedure is a research protocol, have the patient sign any necessary consent form.

4. Fill a 20-ml syringe with normal saline and attach to saline filled extension tubing (35-in.). Obtain intravenous (i.v.) access utilizing a 22-G angiocatheter and attach saline-prepared extension tubing and syringe. This will allow you to flush the extension tubing while the patient waits to be imaged, and, in cases in which a power injector is not available, this will allow preparation for bolus injection (see Note below). Secure the position of the angiocatheter with tegraderm or tape.

Obtaining i.v. access prior to entering the scan room will promote patient throughput and eliminate "dead" time of starting the i.v. while the patient is on the exam table. Follow power injector manufacturer guidelines with regard to appropriate gauge needle/angiocatheter to be used; this will depend on chosen injection/flow rates.

NOTE: If you do not have access to a power injector, you will still be able to perform dynamic imaging as the extension tubing will allow the saline syringe to be placed at the foot of the patient table during pre-contrast imaging. In this case, you will need to draw up the contrast agent in another syringe. When you are ready to bolus inject the contrast agent, simply disconnect the saline syringe and connect the syringe filled with contrast agent; once you have injected the bolus of contrast agent, reconnect the saline syringe and bolus an appropriate volume of flush, usually $\sim 10 \mathrm{ml}$. Alternatively, to eliminate the need of switching syringes, incorporate the use of a 3-way stopcock.

5. Set up the exam room by securing the circularly polarized (CP)-body array coil onto the table and providing a clean exam table.

6. Set up the power injector as specified by the manufacturer.

A minimum of $40 \mathrm{ml}$ normal saline should be drawn up to ensure sufficient saline is available to keep the vein open (KVO) throughout the exam. To determine the amount of contrast agent to be used, reference the contrast agent packet insert and draw up the amount indicated per kg of patient weight. There is no need to double dose.

MRI of the Pancreas

A18.1.2
7. Escort the patient to the MR examination room and ask the patient to lie down accordingly with respect to the exam to be performed. Help the patient mount onto 
the table. Either before or right after the patient lies down, set up any triggering devices or other monitoring equipment that is to be used.

8. Connect the extension tubing secured to the syringe to the power injector extension tubing.

9. Inform the patient about what will occur during the procedure, what he or she will experience while in the magnet, and how to behave, including the following:

a. If earphones or headphones are used to protect the ears from the loud sounds produced by the gradients, the patient will be asked to wear these, but will be able to communicate with you at any time during the imaging.

b. The patient will be given a safety squeeze-bulb or similar equipment to request assistance at any time (demonstrate how this works).

c. For good results the patient should not talk, and should avoid or minimize swallowing or other movement, during each scan-i.e., as long as the banging sounds continue. Between scans, talking and swallowing are allowed in most cases, but should be avoided when comparative positional studies are being performed; the patient will be informed when this is the case. Explain to the patient that one should not reposition one's body between imaging sequences.

Additionally, review breath-holding instructions with the patient, and provide the patient with an approximate time that the examination will take.

d. Nevertheless, the patient may call out at any time if he or she feels it necessary.

e. Position a support under the patient's knees to enhance patient comfort.

10. Secure the top portion of the CP-body array coil to prevent it from moving side-to-side during breath-holding imaging sequences.

Usually straps are provided by the manufacturer that are directly attached to the coil.

11. Use the laser light to position the patient, and to center the coil (see Table A18.1.2). Then advance the patient table to isocenter.

12. If the patient is unable to hold still, provide an appropriate sedative.

13. Program the power injector for a contrast agent and saline injection rate of $2 \mathrm{ml} / \mathrm{sec}$. Total volume of saline following contrast agent injection should be programmed for $10 \mathrm{ml}$. Program a scan delay of $18 \mathrm{sec}$ (contrast agent is injected, scan is initiated 18 sec after contrast agent and saline are delivered). Arm the power injector and keep the vein open.

Do not inject the contrast agent at this time!

\section{Sequence 1: Three-plane positioning scout}

14. To validate the patient's position and to have a reference to prescribe successive imaging sequences, acquire a three-plane orthogonal scout sequence. See Table A18.1.2 for specific parameters.

Most MR scanners can be programmed to acquire the scout automatically after coil tuning or after the patient has been placed in isocenter (for systems that do not require tuning).

\section{Sequence 2: Breath-hold three-plane positioning scout}

15. To have a reference to prescribe successive breath-hold imaging sequences, acquire a second three-plane orthogonal scout sequence. See Table A18.1.2 for specific parameters. 
Table A18.1.2 Imaging Parameters for Scout Sequence (Sequences 1 and 2)

\begin{tabular}{|c|c|}
\hline Patient position & Supine \\
\hline Scan type & Gradient echo \\
\hline Imaging plane (orientation) & Sagittal, transverse, and coronal \\
\hline Central slice or volume center & $\begin{array}{l}\text { Laser light centered } \\
\text { approximately one hand width } \\
\text { above the inferior rib margin }\end{array}$ \\
\hline Echo time $\left(T_{\mathrm{E}}\right)$ & $6 \mathrm{msec}$ \\
\hline Repeat time $\left(T_{\mathrm{R}}\right)$ & $15 \mathrm{msec}$ \\
\hline Flip angle (FA) & $30^{\circ}$ \\
\hline Field of view $\left(\mathrm{FOV}_{x}, \mathrm{FOV}_{y}\right)$ & $450 \mathrm{~mm}, 450 \mathrm{~mm}$ \\
\hline Resolution $\left(\Delta_{x}, \Delta_{y}\right)$ & $1.76 \mathrm{~mm}, 3.52 \mathrm{~mm}$ \\
\hline Number of data points collected $\left(N_{\mathrm{x}}, N_{\mathrm{y}}\right)$ & 256,128 \\
\hline Display matrix $\left(D_{\mathrm{x}}, D_{\mathrm{y}}\right)$ & 256,256 \\
\hline Slice thickness $(\Delta z)$ & $10 \mathrm{~mm}$ \\
\hline Number of slices & 3 \\
\hline Slice gap & Not applicable \\
\hline Number of acquisitions $\left(N_{\text {acq }}\right)$ & 1 \\
\hline Swap read and phase encoding & No \\
\hline Slice locations & Not applicable \\
\hline Saturation pulse & Not applicable \\
\hline Scan time & $16 \mathrm{sec}$ \\
\hline
\end{tabular}

16. Instruct the patient to take in a deep breath and exhale, take in another deep breath and hold it.

17. Initiate the scan.

\section{Sequence 3: Half-acquisition (partial Fourier) turbo spin echo coronal}

18. Display both the coronal and transverse scout images (use non-breath-hold images) in two separate quadrants on the scan monitor. Change imaging parameters to those listed in Table A18.1.3. Position slices to center of the transverse scout, ensuring that the pancreas and liver are covered.

19. Instruct the patient to remain motionless and to breathe normally as the scan will begin and last for $\sim 40 \mathrm{sec}$.

\section{Sequence 4: Half-acquisition turbo spin echo transverse (Fig. A18.1.1)}

20. Display both the coronal and transverse scout images (use non-breath-hold images) in two separate quadrants on the scan monitor. Change imaging parameters to those listed in Table A18.1.4. Position slices to center of the coronal scout, ensuring that the pancreas and liver are entirely covered.

21. Instruct the patient to remain motionless and to breathe normally as the scan will begin and last for $\sim 40 \mathrm{sec}$.

\section{Sequence 5: Transverse gradient echo with fat saturation (fat suppression) (Fig.}

A18.1.2)

22. Display both the coronal and transverse scout images (use breath-hold images) in two separate quadrants on the scan monitor. Change imaging parameters to those listed in Table A18.1.5. Position slices to center of the coronal scout, ensuring that the pancreas is entirely covered. 
It is imperative that the slices are prescribed off of the breath-hold scout images as this is a breath-held imaging sequence. Otherwise, the slice location will not be accurate relative to the reference image if a non-breath-held image is used.

23. Instruct the patient to take in a deep breath and exhale, take in another deep breath and hold it.

24. Initiate the scan.

Table A18.1.3 Imaging Parameters for Half-Acquisition Turbo Spin Echo (Sequence 3)

\begin{tabular}{|c|c|}
\hline Patient position & Supine \\
\hline Scan type & Half-acquisition turbo spin echo \\
\hline Imaging plane (orientation) & Coronal \\
\hline Central slice or volume center & $\begin{array}{l}\text { Slices posted on transverse scout; } \\
\text { center to pancreas }\end{array}$ \\
\hline Echo time $\left(T_{\mathrm{E}}\right)$ & $90 \mathrm{msec}$ \\
\hline Repeat time $\left(T_{\mathrm{R}}\right)$ & $\begin{array}{l}4.4 \mathrm{msec} \text { (note: The true } T_{\mathrm{R}} \text { is } \\
\text { infinite; } 4.4 \mathrm{msec} \text { represents the } \\
\text { echo spacing) }\end{array}$ \\
\hline Delay time $\left(T_{\mathrm{D}}\right)$ & $1500 \mathrm{msec}$ \\
\hline Flip angle (FA) & $150^{\circ}$ \\
\hline Fields of view $\left(\mathrm{FOV}_{x} \mathrm{FOV}_{y}\right)$ & $400 \mathrm{~mm}, 400 \mathrm{~mm}$ \\
\hline Resolution $\left(\Delta_{x} \Delta_{y}\right)$ & $1.56 \mathrm{~mm}, 2.08 \mathrm{~mm}$ \\
\hline Number of data points collected $\left(N_{\mathrm{x}}, N_{\mathrm{y}}\right)$ & 256, 192 (using half Fourier) \\
\hline Display matrix $\left(D_{x}, D_{y}\right)$ & 256,256 \\
\hline Slice thickness $(\Delta z)$ & $8-10 \mathrm{~mm}$ \\
\hline Number of slices & 20 \\
\hline Slice gap & $1.6-2 \mathrm{~mm}$ \\
\hline Number of acquisitions $\left(N_{\text {acq }}\right)$ & 1 \\
\hline Swap read and phase encoding & No \\
\hline Slice location & $\begin{array}{l}\text { Centered to cover the pancreas } \\
\text { and liver }\end{array}$ \\
\hline Saturation pulses & No \\
\hline Slice series & Interleaved \\
\hline Scan time & $40 \mathrm{sec}$ \\
\hline
\end{tabular}

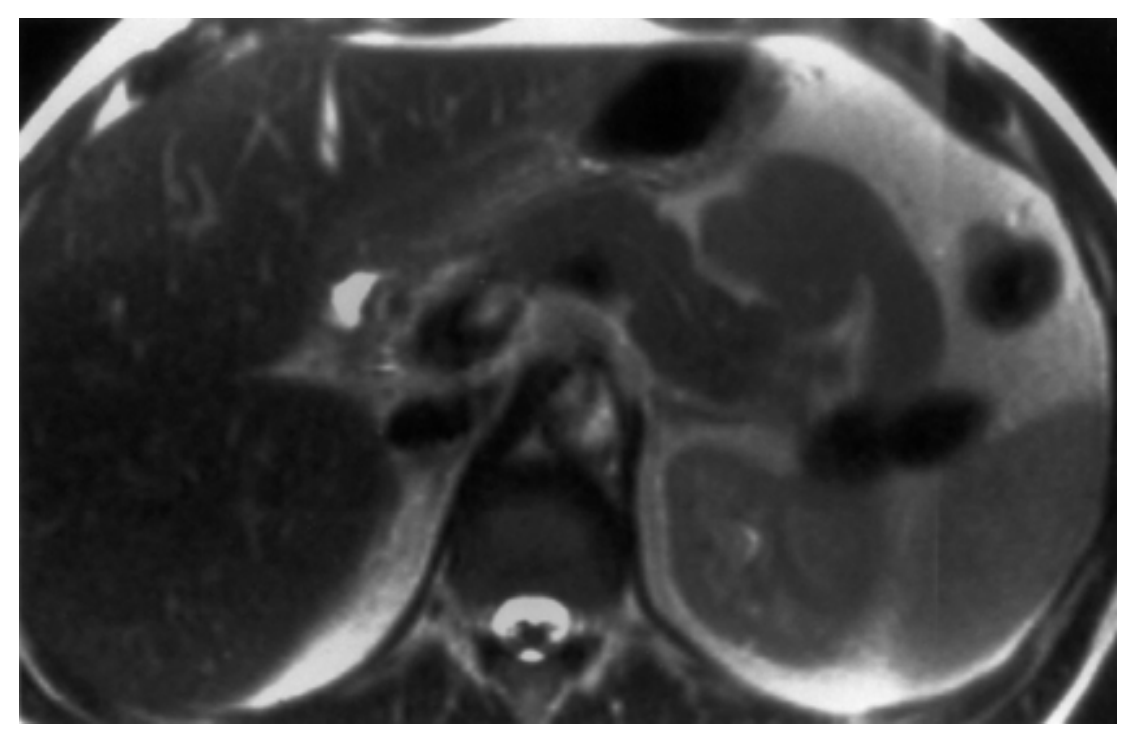

Figure A18.1.1 Unenhanced transverse half-acquisition turbo spin echo image. 
Table A18.1.4 Imaging Parameters for Half-Acquisition Turbo Spin Echo (Sequence 4)

Patient position

Scan type

Imaging plane (orientation)

Central slice or volume center

Echo time $\left(T_{\mathrm{E}}\right)$

Repeat time $\left(T_{\mathrm{R}}\right)$

Delay time $\left(T_{\mathrm{D}}\right)$

Flip angle (FA)

Fields of view $\left(\mathrm{FOV}_{x}, \mathrm{FOV}_{y}\right)$

Resolution $(\Delta x, \Delta y)$

Number of data points collected $\left(N_{\mathrm{x}}, N_{\mathrm{y}}\right)$

Display matrix $\left(D_{\mathrm{x}}, D_{\mathrm{y}}\right)$

Slice thickness $(\Delta z)$

Number of slices

Slice gap

Number of acquisitions $\left(N_{\text {acq }}\right)$

Swap read and phase encoding

Slice location

Saturation pulses

Fat suppression

Slice series

Scan time
Supine

Half-acquisition turbo spin echo

Transverse

Slices posted on coronal; center to pancreas

$90 \mathrm{msec}$

$4.4 \mathrm{msec}$ (note: The true $T_{\mathrm{R}}$ is infinite; $4.4 \mathrm{msec}$ represents the echo spacing)

$1500 \mathrm{msec}$

$150^{\circ}$

$350 \mathrm{~mm}, 263 \mathrm{~mm}$

$1.37 \mathrm{~mm}, 1.37 \mathrm{~mm}$

256, 192 (using half Fourier)

256,256

$8-10 \mathrm{~mm}$

20

$1.6-2 \mathrm{~mm}$

1

No

Centered to cover the pancreas and liver

Yes, superior and inferior to slices No

Interleaved

$40 \mathrm{sec}$

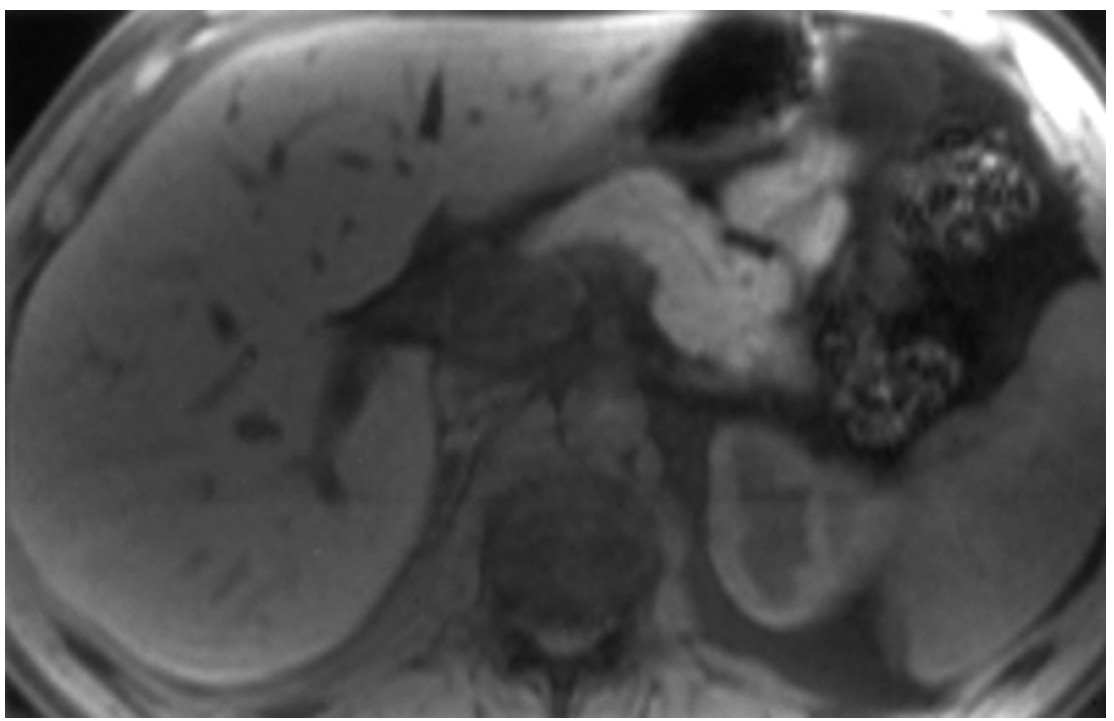

Figure A18.1.2 Unenhanced fat suppressed transverse spoiled gradient echo image. 
Sequence 6: Transverse gradient echo (out-of-phase) (Fig. A18.1.3)

25. Display the coronal and transverse breath-hold scout images (sequence 2) in two separate quadrants on the scan monitor. Change imaging parameters to those listed in Table A18.1.6. Position slices to cover the pancreas and liver.

It is imperative that the slices bee prescribed off of the breath-hold coronal scout as this is a breath-held imaging sequence. Otherwise, the slice location will not be accurate relative to the reference image if a non-breath-held image is used.

Table A18.1.5 Imaging Parameters for Gradient Echo with Fat Saturation (Sequence 5)

\begin{tabular}{ll}
\hline Patient position & Supine \\
Scan type & Gradient echo \\
Imaging plane (orientation) & Transverse \\
Central slice or volume center & Slices posted on coronal; center to \\
& pancreas \\
Echo time $\left(T_{\mathrm{E}}\right)$ & $4.1 \mathrm{msec}$ \\
Repeat time $\left(T_{\mathrm{R}}\right)$ & $147.2 \mathrm{msec}$ \\
Flip angle $(\mathrm{FA})$ & $80^{\circ}$ \\
Fields of view $\left(\mathrm{FOV}, \mathrm{F}_{\mathrm{x}}, \mathrm{F}_{\mathrm{y}}\right)$ & $350 \mathrm{~mm}, 263 \mathrm{~mm}$ \\
Resolution $(\Delta x, \Delta y)$ & $1.37 \mathrm{~mm}, 2.05 \mathrm{~mm}$ \\
Number of data points collected & \\
$\left(N_{\mathrm{x}}, N_{\mathrm{y}}\right)$ & 256,128 \\
Display matrix $\left(D_{\mathrm{x}}, D_{\mathrm{y}}\right)$ & 256,256 \\
Slice thickness $(\Delta z)$ & $6 \mathrm{~mm}$ \\
Number of slices & 20 \\
Slice gap & $1.2 \mathrm{~mm}$ \\
Number of acquisitions $\left(N_{\mathrm{acq}}\right)$ & 1 \\
Swap read and phase encoding & No \\
Slice location & Centered to cover the pancreas \\
Saturation pulses & No \\
Fat suppression & Yes \\
Slice series & Interleaved \\
Scan time & $19 \mathrm{sec}$ \\
\hline &
\end{tabular}

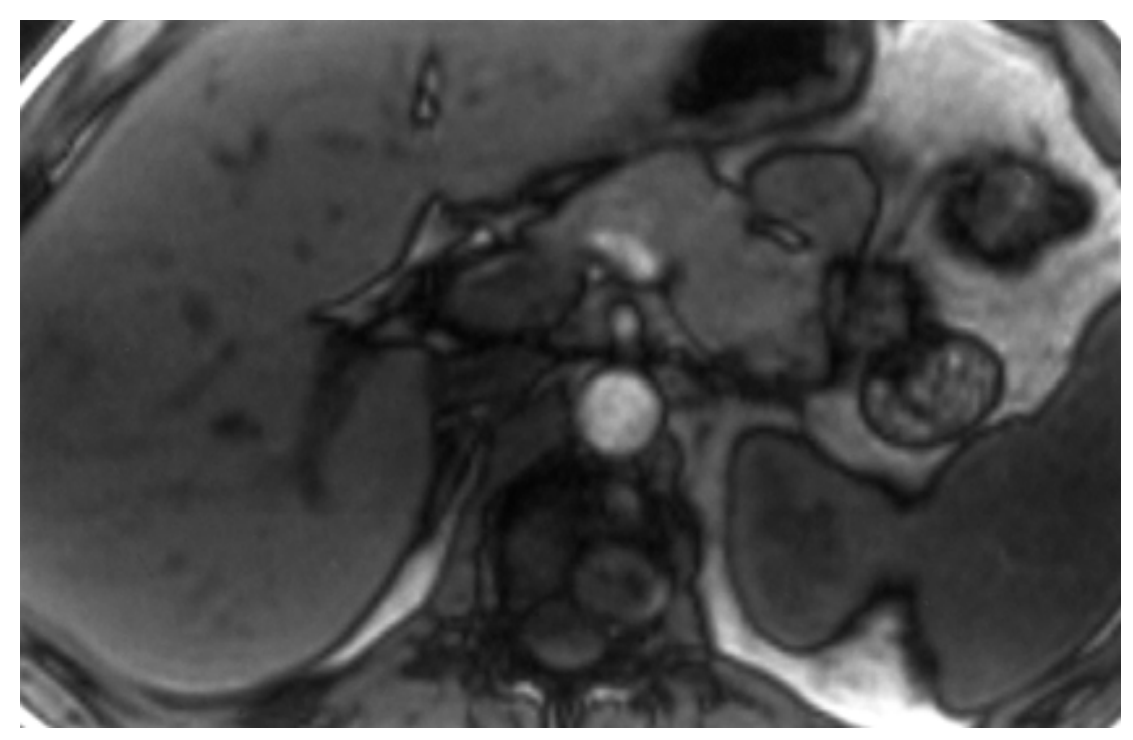

Figure A18.1.3 Out-of-phase transverse spoiled gradient echo image. 
26. Instruct the patient to take in a deep breath and exhale, take in another deep breath and hold it.

27. Initiate the scan.

Sequence 7: Transverse gradient echo (Fig. A18.1.4)

28. Display the breath-hold coronal and transverse scout images (sequence 2) in two separate quadrants on the scan monitor. Change imaging parameters to those listed in Table A18.1.7. Position slices to cover the pancreas and liver.

Table A18.1.6 Imaging Parameters for Gradient Echo-Out-of-Phase (Sequence 6)

\begin{tabular}{ll}
\hline Patient position & Supine \\
Scan type & Gradient echo \\
Imaging plane (orientation) & Transverse \\
Central slice or volume center & $\begin{array}{l}\text { Slices posted on coronal; center to } \\
\text { the pancreas }\end{array}$ \\
Echo time $\left(T_{\mathrm{E}}\right)$ & $2.2 \mathrm{msec}$ \\
Repeat Time $\left(T_{\mathrm{R}}\right)$ & $140 \mathrm{msec}$ \\
Flip angle $(\mathrm{FA})$ & $80^{\circ}$ \\
Fields of view $(\mathrm{FOV}$ & $\mathrm{x}, \mathrm{FOV} \mathrm{y})$ \\
Resolution $(\Delta x, \Delta y)$ & $350 \mathrm{~mm}, 263 \mathrm{~mm}$ \\
Number of data points collected $\left(N_{\mathrm{x}}, N_{\mathrm{y}}\right)$ & $1.37 \mathrm{~mm}, 2.05 \mathrm{~mm}$ \\
Display matrix $\left(D_{\mathrm{x}}, D_{\mathrm{y}}\right)$ & 256,128 \\
Slice thickness $(\Delta z)$ & 256,256 \\
Number of slices & $8-10 \mathrm{~mm}$ \\
Slice gap & 20 \\
Number of acquisitions $\left(N_{\mathrm{acq}}\right)$ & $1.6-2 \mathrm{~mm}$ \\
Swap read and phase encoding & 1 \\
Slice location & No \\
Saturation pulses & Centered to cover the pancreas \\
Slice series & and liver \\
Scan time & No \\
\hline
\end{tabular}

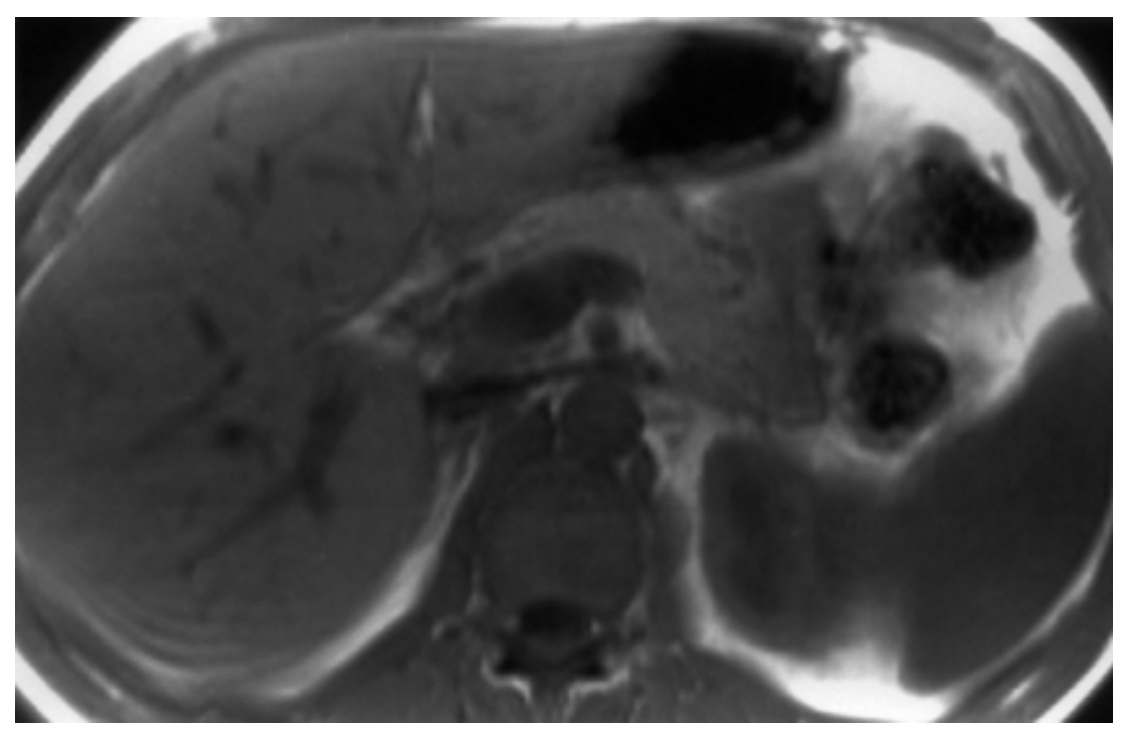

Figure A18.1.4 Unenhanced transverse spoiled gradient echo image. 


\begin{tabular}{ll}
\hline Patient position & Supine \\
Scan type & $\begin{array}{l}\text { Gradient echo } \\
\text { Transverse }\end{array}$ \\
Central slice or volume center & Slices posted on coronal; center to \\
& the pancreas \\
Echo time $\left(T_{\mathrm{E}}\right)$ & $4.1 \mathrm{msec}$ \\
Repeat time $\left(T_{\mathrm{R}}\right)$ & $140 \mathrm{msec}$ \\
Flip angle $(\mathrm{FA})$ & $80^{\circ}$ \\
Fields of view $\left(\mathrm{FOV}, \mathrm{F}_{\mathrm{x}}\right.$, FOV & \\
Resolution $(\Delta x, \Delta y)$ & $350 \mathrm{~mm}, 263 \mathrm{~mm}$ \\
Number of data points collected $\left(N_{\mathrm{x}}, N_{\mathrm{y}}\right)$ & $1.37 \mathrm{~mm}, 2.05 \mathrm{~mm}$ \\
Display matrix $\left(D_{\mathrm{x}}, D_{\mathrm{y}}\right)$ & 256,128 \\
Slice thickness $(\Delta z)$ & 256,256 \\
Number of slices & $8-10 \mathrm{~mm}$ \\
Slice gap & 18 \\
Number of acquisitions $\left(N_{\mathrm{acq}}\right)$ & $1.6-2 \mathrm{~mm}$ \\
Swap read and phase encoding & 1 \\
Slice location & No \\
Saturation pulses & Centered to cover the pancreas \\
Slice series & and liver \\
Scan time & No \\
\hline & Interleaved \\
& $18 \mathrm{sec}$ \\
\hline
\end{tabular}

It is imperative that the slices are prescribed off of the breath-hold coronal scout image as this is a breath-held imaging sequence. Otherwise, the slice location will not be accurate relative to the reference image if a non-breath-held image is used.

29. Instruct the patient to take in a deep breath and exhale, take in another deep breath and hold it.

30. Initiate the scan.

Sequence 8: Transverse gradient echo-Immediate postcontrast (Fig. A18.1.5) NOTE: See patient set up section for specific instructions on preparation for contrast injection. This preparation must be done prior to placing the patient in the scanner.

31. Repeat step 28 .

It is imperative that the slices be prescribed off of the breath-hold coronal scout image as this is a breath-held imaging sequence. Otherwise, the slice location will not be accurate relative to the reference image if a non-breath-held image is used.

32. Explain to the patient that you will now be injecting the contrast agent and he/she may feel a cool sensation in his/her arm. Initiate the injection. Do not begin scanning until the $18 \mathrm{sec}$ scan delay has expired. However, deliver breathing instructions when $10 \mathrm{sec}$ of delay are remaining (see step 33).

If you do not have access to a power injector and are "hand" injecting, you can still use step 32. However, after you have completed the bolus contrast injection, reattach the saline-filled syringe and flush with $10 \mathrm{ml}$ of saline. Begin breathing instructions after $5 \mathrm{ml}$ of the saline has been injected, then proceed to initiate the scan. The process of switching syringes must be completed as quickly as possible and thus it is suggested that a 3-way stopcock be incorporated. 


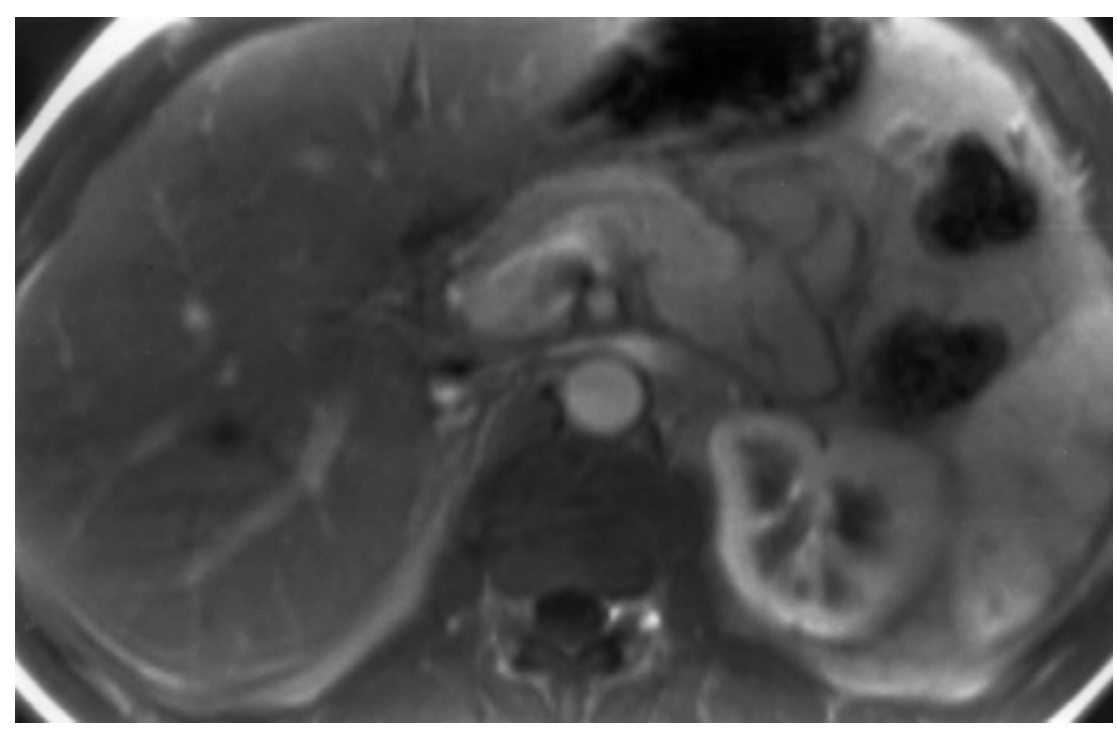

Figure A18.1.5 Immediate post contrast transverse spoiled gradient echo image.

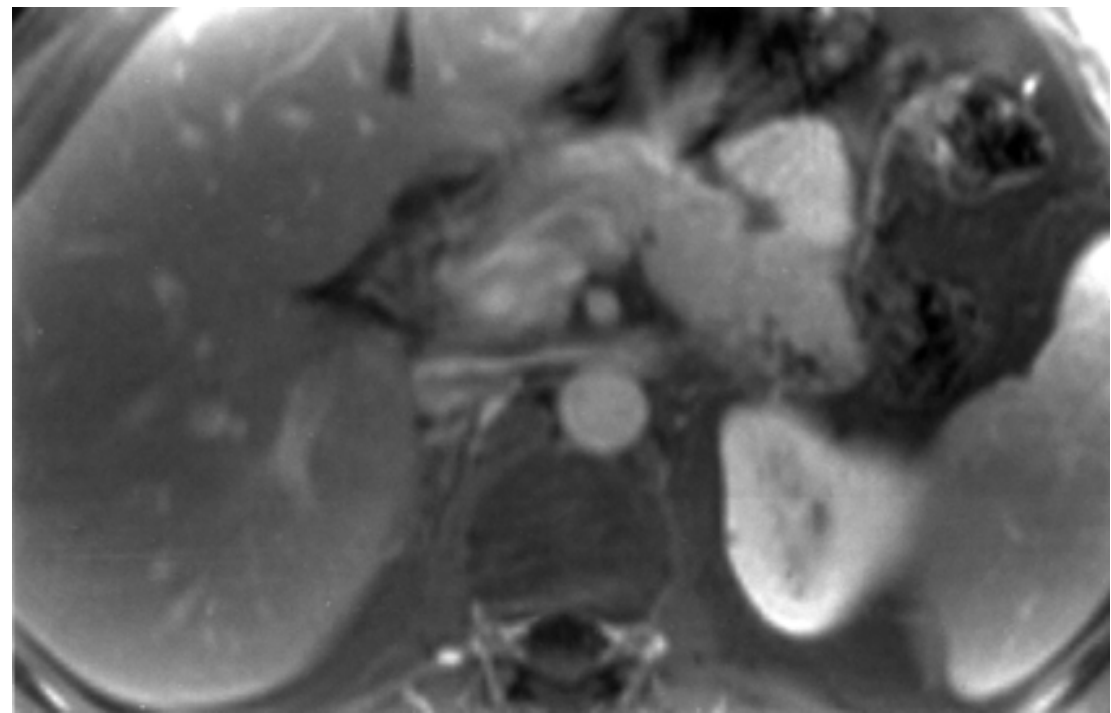

Figure A18.1.6 Post contrast fat suppressed transverse spoiled gradient echo image.

33. When there are $10 \mathrm{sec}$ of delay remaining, instruct the patient to take in a deep breath and exhale, take in another deep breath and hold it.

34. Initiate the scan.

\section{Sequence 9: Transverse gradient echo-45 sec delay after sequence 8}

35. Repeat step 28.

36. Once $45 \mathrm{sec}$ has expired, instruct the patient to take in a deep breath and exhale, take in another deep breath and hold it.

37. Initiate the scan.

\section{Sequence 10: Transverse gradient echo with fat saturation (90 sec delay after} injection; Fig. A18.1.6)

38. Display the midline slice of the breath-hold coronal and transverse scout images (sequence 2) in two separate quadrants on the scan monitor. Change imaging 
Table A18.1.8 Imaging Parameters for Gradient Echo with Fat Saturation-90

Sec Delay (Sequence 10)

$\begin{array}{ll}\text { Patient position } & \begin{array}{l}\text { Supine } \\ \text { Gradient echo } \\ \text { Scan type }\end{array} \\ \text { Imaging plane (orientation) } & \begin{array}{l}\text { Sransverse } \\ \text { the pancreas }\end{array} \\ \text { Central slice or volume center } & 4.1 \mathrm{msec} \\ & 147.2 \mathrm{msec} \\ \text { Echo time }\left(T_{\mathrm{E}}\right) & 80^{\circ} \\ \text { Repeat time }\left(T_{\mathrm{R}}\right) & 350 \mathrm{~mm}, 263 \mathrm{~mm} \\ \text { Flip angle }(\mathrm{FA}) & 1.37 \mathrm{~mm}, 2.05 \mathrm{~mm} \\ \text { Fields of view }\left(\mathrm{FOV}_{\mathrm{x}}, \mathrm{FOV}_{\mathrm{y}}\right) & 256,128 \\ \text { Resolution }(\Delta x, \Delta y) & 256,256 \\ \text { Number of data points collected }\left(N_{\mathrm{x}}, N_{\mathrm{y}}\right) & 6 \mathrm{~mm} \\ \text { Display matrix }\left(D_{\mathrm{x}}, D_{\mathrm{y}}\right) & 20 \\ \text { Slice thickness }(\Delta z) & 1.2 \mathrm{~mm} \\ \text { Number of slices } & 1 \\ \text { Slice gap } & \text { No } \\ \text { Number of acquisitions }\left(N_{\mathrm{acq}}\right) & \text { Centered to cover entire pancreas } \\ \text { Swap read and phase encoding } & \text { and liver } \\ \text { Slice location } & \text { No } \\ & \text { Yes } \\ \text { Saturation pulses } & \text { Interleaved } \\ \text { Fat suppression } & 19 \text { sec } \\ \text { Slice series } & \end{array}$

parameters to those listed in Table A18.1.8. Position slices to cover the pancreas and liver.

It is imperative that the slices are prescribed off of the gradient echo coronal image as this is a breath-held imaging sequence. Otherwise, the slice location will not be accurate relative to the reference image if a non-breath-held image is used.

39. Once $90 \mathrm{sec}$ has expired, instruct the patient to take in a deep breath and exhale, then take in another deep breath and hold it.

40. Initiate the scan.

\section{COMMENTARY}

\section{Background Information}

MRI using a combination of non-contrast $T_{1}$-weighted fat suppressed imaging and immediate post-gadolinium spoiled gradient echo has been shown to be effective at exhibiting the full range of pancreatic disease (Gabata et al., 1994; Kelekis et al., 1995; Mitchell et al., 1995; Semelka et al., 1991; Semelka and Ascher, 1993; Semelka et al., 1993a,b; Semelka et al., 1996; Winston et al., 1995). Normal pancreas enhances in a moderately intense, uniform fashion on immediate post-gadolinium images, reflecting a uniform network of capillary vessels (Semelka et al., 1991; Semelka and Ascher, 1993). The normal pancreas is also moderately intense on non-contrast $T_{1}$-weighted fat suppressed images due to the presence of aqueous protein in the ascini of the glandular pancreas (Mitchell et al., 1995; Semelka et al., 1991; Semelka et al., 1993a; Winston et al., 1995). Particular strengths of MRI include the detection of small ductal adenocarcinomas and islet cell tumors (Gabata et al., 1994; Kelekis et al., 1995; Semelka et al., 1991; Semelka et al., 1993a,b; Semelka et al., 1996).

\section{Critical Parameters and} Troubleshooting

The most important data acquisition is the immediate post gadolinium spoiled gradient
Pancreas

A18.1.11 
Table A18.1.9 Imaging Parameters for Breathing Averaged Fat Suppressed Spin Echo (Use if Fat Suppressed Spoiled Gradient Echo Sequence Is Unavailable)

\begin{tabular}{ll}
\hline Patient position & Supine \\
Scan type & Spin echo \\
Imaging plane (orientation) & Transverse \\
Central slice or volume center & Slices posted on coronal; center to \\
& pancreas \\
Echo time $\left(T_{\mathrm{E}}\right)$ & $15 \mathrm{msec}$ \\
Repeat time $\left(T_{\mathrm{R}}\right)$ & $500 \mathrm{msec}$ \\
Flip angle $(\mathrm{FA})$ & $90^{\circ}$ \\
Fields of view $\left(\mathrm{FOV}_{\mathrm{x}}, \mathrm{FOV}_{\mathrm{y}}\right)$ & $350 \mathrm{~mm}, 263 \mathrm{~mm}$ \\
Resolution $(\Delta x, \Delta y)$ & $1.37 \mathrm{~mm}, 2.05 \mathrm{~mm}$ \\
Number of data points collected $\left(N_{\mathrm{x}}, N_{\mathrm{y}}\right)$ & 256,128 \\
Display matrix $\left(D_{\mathrm{x}}, D_{\mathrm{y}}\right)$ & 256,256 \\
Slice thickness $(\Delta z)$ & $8-10 \mathrm{~mm}$ \\
Number of slices & 10 \\
Slice gap & $1.6-2 \mathrm{~mm}$ \\
Number of acquisitions $\left(N_{\text {acq }}\right)$ & 4 \\
Swap read and phase encoding & No \\
Slice location & Centered to cover pancreas \\
Saturation pulses & No \\
Fat suppression & Yes \\
Slice series & Interleaved \\
Scan time & 4 min, 19 sec \\
\hline
\end{tabular}

echo sequence. The critical aspect of this sequence is that the timing of gadolinium must be during the hepatic arterial dominant phase (or capillary phase) of enhancement (Semelka et al., 1993a). To ensure appropriate timing, contrast should be present in the hepatic arteries and portal veins and not yet present in the hepatic veins (Semelka et al., 1993a). The patient must be able to breath-hold for $20 \mathrm{sec}$ to achieve optimal results for studying the pancreas, as required for the $T_{1}$-weighted gradient echo sequence.

Fat-suppressed spin echo (see Table A18.1.9) can be substituted for the fat suppressed spoiled gradient echo, if this latter sequence is not available on the scanner. Patients who are unable to breathhold or breathe in a regular fashion are better examined by $\mathrm{CT}$ (computed tomography).

\section{Anticipated Results}

In a patient who can suspend respiration and using an MR protocol that includes non-contrast $T_{1}$-weighted fat suppressed spoiled gradient echo and immediate post contrast spoiled gradient echo imaging, one can successfully evaluate a full range of pancreatic diseases.
Particular strengths of this approach are in the detection of small pancreatic ductal adenocarcinomas, ampullary tumors and islet cell tumors, and staging of pancreatic neoplasms in general.

\section{Literature Cited}

Gabata, T., Matsui, O., Kadoya, M., Yoshikawa, J., Miyayama, S., Takashima, T., Nagakawa, T., Kayahara, M., and Nonomura, A. 1994. Small pancreatic adenocarcinomas: Efficacy of MR imaging with fat suppression and gadolinium enhancement. Radiology 193:683-688.

Kelekis, N.L., Semelka, R.C., Molina, P.L., and Doer, M.E. 1995. ACTH-secreting islet cell tumor: Appearances on dynamic gadolinium-enhanced MRI. J. Magn. Reson. Imag. 13:641.

Mitchell, D.G., Winston, C.B., Outwater, E.K., and Ehrlich S.M. 1995. Delineation of pancreas with MR imaging: Multi-observer comparison of five pulse sequences. J. Magn. Reson. Imag. 5:193199.

Semelka, R.C. and Ascher, S.M. 1993. MRI of the pancreas: State of the art. Radiology 188:593602.

Semelka, R.C., Kroeker, M.A., Shoenut, J.P., Kroeker, R., Yaffe, C.S., and Micflikier, A.B. 1991. Pancreatic disease: prospective comparison of CT, ERCP, and 1.5T imaging with dy- 
namic gadolinium enhancement and fat suppression. Radiology181:785-791.

Semelka, R.C., Cummings, M., Shoenut, J.P., Yaffe, C.S., Kroeker, M.A., and Greenberg, H.M. 1993a. Islet cell tumors: A comparison of detection by dynamic contrast-enhanced CT and MR imaging with dynamic gadolinium enhancement and fat suppression. Radiology186:799-802.

Semelka, R.C., Shoenut, J.P., Kroeker, M.A., and Micflikier, A.B. 1993b. Chronic pancreatitis: MR imaging features before and after administration of gadopentetate dimeglumine. J. Magn. Reson. Imag. 3:79-82.

Semelka, R.C., Kelekis, N.L., Molina, P.L., Sharp, T., and Calvo, B. 1996. Pancreatic masses with indeterminate findings on spiral CT, Is there a role for MRI? J. Magn. Reson. Imag. 6:585-588.

Shellock, F.G. 1996. Pocket Guide to MR Procedures and Metallic Objects. Lippincott-Raven, Philadelphia.

Winston, C.B., Mitchell, D.G., Outwater, E.K., and Ehrlich, S.M. 1995. Pancreatic signal intensity on $T_{1}$-weighted fat saturation MR images: Clinical correlation. J. Magn. Reson. Imag. 5:267271.

\section{Key References}

Shellock, F.G. 1996.

Covers a number of important patient management issues related to MR imaging, including recommended safety procedures, a list of metallic implants that have been tested for MR compatibility, and a list of other sources on MR safety.

\section{Contributed by Kathy Wilber and \\ Richard Semelka \\ University of North Carolina-Chapel Hill Chapel Hill, North Carolina}

Laurie Fisher

Siemens Uptime Service Center

Cary, North Carolina 
